\title{
Correction to: Exploring the Relationship between Character Strengths and Meditation: a Cross-Sectional Study among Long-Term Practitioners of Sahaja Yoga Meditation
}

\section{Tommy Hendriks ${ }^{1} \cdot$ Joshua Pritikin ${ }^{2} \cdot$ Rajeev Choudhary ${ }^{3} \cdot$ Chad Danyluck $^{4}$}

Published online: 6 July 2021

(C) The Author(s) 2021

\section{Correction to: International Journal of Applied Positive Psychology https://doi.org/10.1007/s41042-021-00052-0}

In the initial online publication of this article the presentation of the Appendix 1 was incorrect and Appendix 2 was missing. The correct versions of Appendices 1 and 2 are given below. The original article has been corrected.

The online version of the original article can be found at https://doi.org/10.1007/s41042-021-00052-0

Tommy Hendriks

t.hendriks_2@tilburguniversity.edu

1 Department of Human Resource Studies \& Department of Developmental Psychology, Tilburg University, Warandelaan 2, 5037 ABTilburg, the Netherlands

2 Virginia Institute for Psychiatric and Behavioral Genetics, Virginia Commonwealth University, Richmond, VA, USA

3 Pt. Ravishankar Shukla University, Raipur, Chhattisgarh, India

4 Department of Psychology, Carleton University, Ottawa, Ontario, Canada 


\section{Appendix 1 Emic psycho-physiological strength model of Sahaja Yoga meditation}

Here is a possible assignment of character strengths to SY qualities. Some effort was undertaken to assign each character strength to a single quality most closely associated with SY, but true assignments may not be so constrained. This chart should be regarded as provisional and subject to revision.

\begin{tabular}{|c|c|c|c|c|c|}
\hline Center/Chakra & SY Qualities & $\begin{array}{l}\text { Character } \\
\text { strengths }\end{array}$ & Center/Chakra & SY Qualities & $\begin{array}{l}\text { Character } \\
\text { strengths }\end{array}$ \\
\hline $\begin{array}{l}\text { Mooladhara } \\
\qquad \text { (Pelvis plexus) }\end{array}$ & $\begin{array}{l}\text { - Innocence } \\
\text { - Wisdom } \\
\text { - Chastity/Purity } \\
\text { - Eternal Childhood }\end{array}$ & - Curiosity & $\begin{array}{l}\text { Anahata (Centre } \\
\text { cardiac plexus) }\end{array}$ & $\begin{array}{l}\text { - Love } \\
\text { - Compassion } \\
\text { - Security } \\
\text { - Fearlessness } \\
\text { - Courage } \\
\text { - Responsibility }\end{array}$ & $\begin{array}{l}\text { - Love } \\
\text { - Bravery } \\
\text { - Perseverance }\end{array}$ \\
\hline $\begin{array}{l}\text { Swadisthan } \\
\qquad \text { (Aortic plexus) }\end{array}$ & $\begin{array}{l}\text { - Creativity } \\
\text { - Attention/Knowledge } \\
\text { - Inspiration } \\
\text { - Energy } \\
\text { - Sense of Aesthetics } \\
\quad \text { and Art }\end{array}$ & $\begin{array}{l}\text { - Creativity } \\
\text { - Love } \\
\text { of Learning } \\
\text { - Zest } \\
\text { - Appreciation of } \\
\text { Beauty and } \\
\text { Excellence }\end{array}$ & $\begin{array}{l}\text { Vishuddhi } \\
\quad \text { (Cervical plexus) }\end{array}$ & $\begin{array}{l}\text { - Collectivity } \\
\text { - Communication } \\
\text { - Truthfulness } \\
\text { - Diplomacy } \\
\text { - Detachment } \\
\text { - (Self) Respect }\end{array}$ & $\begin{array}{l}\text { - Teamwork } \\
\text { - Honesty } \\
\text { - Prudence } \\
\text { - Perspective }\end{array}$ \\
\hline Nabhi (Solar plexus) & $\begin{array}{l}\text { - Satisfaction } \\
\text { - Peacefulness } \\
\text { - Generosity Evolution }\end{array}$ & $\begin{array}{l}\text { - Gratitude } \\
\text { - Fairness }\end{array}$ & $\begin{array}{l}\text { Agnya \& Hamsa } \\
\text { (Crossing optic } \\
\text { thalamus) }\end{array}$ & $\begin{array}{l}\text { - Forgiveness } \\
\text { - Humility } \\
\text { - Discernment }\end{array}$ & $\begin{array}{l}\text { - Forgiveness } \\
\text { - Humility } \\
\text { - Judgment }\end{array}$ \\
\hline Bhavsagar* & $\begin{array}{l}\text { - Self-Mastery } \\
\text { - Balance/Stability } \\
\text { - Righteousness }\end{array}$ & - Self-regulation & $\begin{array}{l}\text { Sahasrara } \\
\qquad \text { (limbic area) }\end{array}$ & $\begin{array}{l}\text { - Joy } \\
\text { - Integration } \\
\quad \text { (Self realization) } \\
\text { - Thoughtless } \\
\text { awareness } \\
\text { - Collective } \\
\text { consciousness }\end{array}$ & Spirituality \\
\hline
\end{tabular}

*Bhavsagar, or void, is a region located within the abdominal cavity. It contains the nabhi 
Appendix 2 Overview of means and standard deviation of VIA-IS character strengths by group

\begin{tabular}{|c|c|c|c|c|c|c|}
\hline Character Strength & Group & Mean & SD & Group & Mean & SD \\
\hline Spirituality & SY & 4.50 & 0.49 & CG & 3.36 & 1.00 \\
\hline Forgiveness & SY & 4.25 & 0.56 & $\mathrm{CG}$ & 3.73 & 0.72 \\
\hline Gratitude & SY & 4.27 & 0.57 & $\mathrm{CG}$ & 3.85 & 0.73 \\
\hline Self-Regulation & SY & 3.70 & 0.62 & CG & 3.32 & 0.79 \\
\hline Teamwork & SY & 4.08 & 0.56 & $\mathrm{CG}$ & 3.78 & 0.67 \\
\hline Appreciation of Beauty & SY & 4.23 & 0.55 & CG & 3.93 & 0.72 \\
\hline Appreciation of Beauty & SY & 4.04 & 0.55 & $\mathrm{CG}$ & 3.73 & 0.75 \\
\hline Kindness & SY & 4.34 & 0.51 & CG & 4.14 & 0.58 \\
\hline Zest & SY & 3.89 & 0.66 & $\mathrm{CG}$ & 3.64 & 0.77 \\
\hline Fairness & SY & 4.28 & 0.53 & CG & 4.11 & 0.58 \\
\hline Judgment & SY & 3.96 & 0.57 & CG & 4.13 & 0.59 \\
\hline Curiosity & SY & 4.12 & 0.60 & CG & 3.91 & 0.72 \\
\hline Love & SY & 4.15 & 0.58 & $\mathrm{CG}$ & 3.96 & 0.69 \\
\hline Humility & SY & 3.71 & 0.66 & $\mathrm{CG}$ & 3.51 & 0.75 \\
\hline Leadership & SY & 4.02 & 0.56 & $\mathrm{CG}$ & 3.85 & 0.64 \\
\hline Bravery & SY & 3.81 & 0.63 & $\mathrm{CG}$ & 3.64 & 0.73 \\
\hline Love of Learning & SY & 3.72 & 0.69 & CG & 3.90 & 0.77 \\
\hline Perseverance & SY & 3.89 & 0.64 & $\mathrm{CG}$ & 3.71 & 0.82 \\
\hline Social Intelligence & SY & 4.00 & 0.56 & $\mathrm{CG}$ & 3.87 & 0.65 \\
\hline Perspective & SY & 3.92 & 0.64 & $\mathrm{CG}$ & 3.81 & 0.65 \\
\hline Prudence & SY & 3.81 & 0.62 & $\mathrm{CG}$ & 3.70 & 0.70 \\
\hline Honesty & SY & 4.42 & 0.45 & CG & 4.34 & 0.49 \\
\hline Humor & SY & 3.88 & 0.67 & $\mathrm{CG}$ & 3.80 & 0.77 \\
\hline Creativity & SY & 3.89 & 0.65 & CG & 3.87 & 0.77 \\
\hline
\end{tabular}

Publisher's Note Springer Nature remains neutral with regard to jurisdictional claims in published maps and institutional affiliations. 